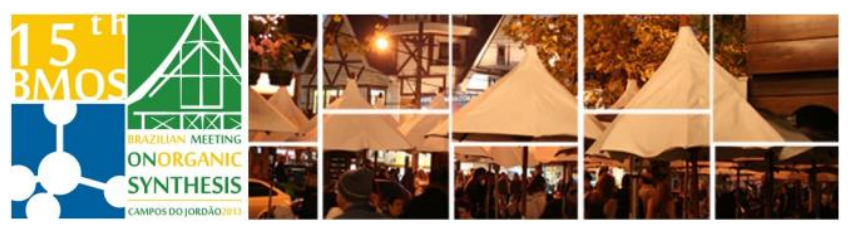

\title{
Strategies for Total Synthesis of Pyrenophorin
}

\author{
Jaqueline Rosa C. Barbosa(PG) ${ }^{1,2 \star}$, Mauricio Moraes Victor(PQ) ${ }^{1,2}$. \\ ${ }^{1}$ Organic Chemistry Department, Chemistry Institute, Federal University of Bahia (UFBA), CEP: 40170-290/ \\ ${ }^{2}$ National Institute of Science \& Technology for Energy and Environment, Federal University of Bahia
}

(UFBA), CEP: 40210-630

*jaquelinerosa.cb@gmail.com:

Keywords: Pyrenophorin, coupling, Nozaki-Hiyama-Kishi

\section{INTRODUCTION}

Molecules belonging to the medium and large size rings lactones have attracted considerable attention from synthetic chemists due to their interesting biological properties. Due these structural features and importance, we have accepted the challenging to prepare Pyrenophorin 1 (Figure 1), a symmetric sixteen-membered diolide isolated from Pyrenophora avenae ${ }^{1}$ which displays potent antifungal activity. In our synthetic strategy to obtain a half-part of this molecule, we did testing by using some methods of coupling between fragments $\mathbf{3}$ and 4 for reach the ester 2 . Further early experiments to obtain a ring intermediate have been made by using Nozaki-Hiyama-Kishi reaction. This work shows our results in this direction.

\section{RESULTS AND DISCUSSION}

Our efforts began with the choice of best silylprotecting group of (+/-)-1,4-pentanediol. Among protected agent that were tested the best results were reached at lower temperature reaction $\left(0{ }^{\circ} \mathrm{C}\right)$ performed with TIPSCl as silylating agent and was obtained $85 \%$ yield.

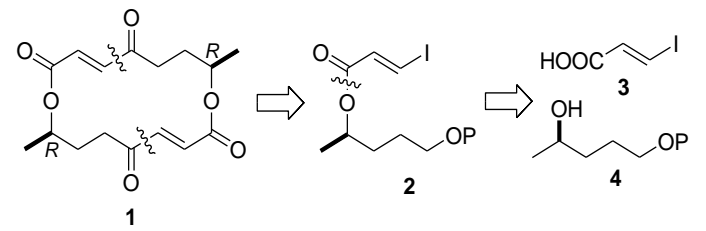

Figure 1. Retrosynthetic analysis.

After preparation of 1-O-protected diols, we decided to investigate a suitable methodology to coupling the monosilylated alcohols $\mathbf{4}$ and E-iodo acrylic acid (3). Among strategies that were tested, those which reached more promising results were: Yamaguhi reaction and Mitsunobu reaction.

We tested 2 different methodologies with Yamaguchi chloride: a classical procedure ${ }^{2}$ where a mixed anhydride was isolated before addition of alcohol; and a one-pot procedure, without isolation of intermediate ${ }^{3}$. The best result obtained was one-pot Yamaguchi methodology that was by using DIPEA as base, toluene during $72 \mathrm{~h}$ at room temperature obtained $48 \%$ yield.

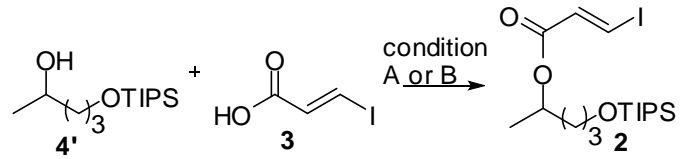

Scheme 1. Coupling- A: Yamaguchi chloride, DIPEA, toluene, $0^{\circ}-$ rt, $48 \%$. B: DIAD, $\mathrm{PPh}_{3}$, THF, 0 --r.t, $32 \%$.

And then was tested the Mitsunobu methodology ${ }^{4}$ by using DIAD, $\mathrm{PPh}_{3}$ in THF during $72 \mathrm{~h}$ at room temperature obtained $32 \%$ yield.<smiles>CC(/C=C/C(O)/C=C/C(=O)OC(C)CCC=O)OC(=O)/C=C/I</smiles>

Scheme 2. Coupling- 1)TBAF, THF, 3h. r.t. 2) $\mathrm{PCC}, \mathrm{CH}_{2} \mathrm{Cl}_{2}$, 4h, r.t. 3) $\mathrm{CrCl}_{2} / \mathrm{Ni}$, DMF. 48 h, r.t.

After obtained the ester intermediate 2' was desprotected with TBAF in THF during $3 \mathrm{~h}$ to formed the alcohol with $75 \%$ yield. In sequence the alcohol was oxidized with PCC in DCM to obtained the aldehyde.

Immediately from aldehyde crude was made early tests by using Nozaki-Hiyama-Kishi reaction with $5 \%$ total yield, and then observed evidence of the formation of an alcohol through infrared spectrum.

\section{CONCLUSION}

Then until now the best methodology to coupling 4 and 3 was Yamaguchi one-pot with $48 \%$ yield, but more investigations will be made. Early test to formation of ring intermediate were promising.

ACKNOWLEDGEMENTS

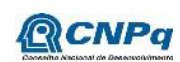

\section{REFERENCES}

${ }^{1}$ Ishibashi, K.; Agric. Chem. Soc. 1961, 35, 257.

2 Inanaga, J.; Hirata, K.; Saeki, H.; Katsuki, T.; Yamaguchi, M.; Bull. Chem. Soc. Jpn. 1979, 52, 1989.

${ }^{3}$ Navarro, I.; Basset, J.-F.; Hebbe, S.; Major, S. M.; Werner, T.; Howsham, C.; Brackow, J.; Barrett, A. G. M.; J. Am. Chem. Soc. 2008, 130, 10293.

${ }^{4}$ Lee, D.; Yun, S.Y.; Hansen, E.C.; Vochkov, I.; Cho, E.J.; Lo, W.Y. Angew. Chem. Int. Ed. 2010, 49, 4263.

\footnotetext{
$15^{\text {th }}$ Brazilian Meeting on Organic Synthesis - 15 $5^{\text {th }}$ BMOS - November 10-13, 2013 - Campos do Jordão, Brazil
} 